\title{
Downward bias of conductivity based point-of- care hemoglobin measurement compared with optical methods
}

\author{
Min Hee Heo', Jun Hyun Kim', Kyung Woo Kim², Ho Jae Cho', \\ Won Joo Choe', Kyung-Tae Kim¹, Ji Yeon Kim', Sang-Il Lee', \\ Jang Su Park', and Jung Won Kim ${ }^{1}$ \\ ${ }^{1}$ Department of Anesthesiology and Pain Medicine, Inje University Ilsan Paik Hospital, \\ Goyang, ${ }^{2}$ Department of Anesthesiology and Pain Medicine, Inje University Seoul Paik \\ Hospital, Seoul, Korea
}

Received September 7, 2017

Revised 1st, November 7, 2017

2nd, November 25, 2017

Accepted November 27, 2017

\section{Corresponding author}

Jun Hyun Kim, M.D.

Department of Anesthesiology and Pain Medicine, Inje University Ilsan Paik Hospital, 170 Juhwa-ro, Ilsanseo-gu, Goyang 10380, Korea Tel: 82-31-910-7067

Fax: 82-31-910-7184

E-mail: ishmael1@naver.com

\section{ORCID}

http://orcid.org/0000-0002-9466-8399
Background: Point-of-care (POC) arterial blood gas analysis (ABGA) is widely used for checking hemoglobin $(\mathrm{Hb})$ level. However, there is the tendency of downward bias of conductivity-based POC ABGA Hb measurement compared with optical methods. Authors tried to correct that bias by linear regression equation.

Methods: We retrospectively collected a total of $86 \mathrm{Hb}$ result pairs during surgeries. $\mathrm{Hb}$ measured by the Sysmex XE-2100 in the laboratory was set as the gold standard and was compared with that measured by the GEM Premier 3500. Data were compared using the Bland-Altman analysis, the reliability of transfusion decision was assessed using three-zone error grid. The linear regression analysis was performed to find out the relation between the $\mathrm{Hb}$ results of POC ABGA and those of laboratory based test.

Results: The bias of the $\mathrm{Hb}$ measured between Sysmex XE-2100 and GEM Premier 3500 was $-0.9 \mathrm{~g} / \mathrm{dl}(\mathrm{P}<0.001,95 \%$ confidence interval, -1.038 to $-0.665 \mathrm{~g} / \mathrm{dl})$. The percentage error was $16.4 \%$. According to error grid methodology, zone A, B and C encompassed $89.5 \%, 10.5 \%$ and $0 \%$ of data pairs. After adjusting the POC ABGA Hb values, the bias of the $\mathrm{Hb}$ measured by two methods was $0 \mathrm{~g} / \mathrm{dl}(\mathrm{P}=0.991)$. The percentage error was $18.2 \%$. The zone A, B and C encompassed $91.9 \%, 8.1 \%$ and $0 \%$ of data pairs.

Conclusions: $\mathrm{Hb}$ measurements obtained with reference to conductivity via a POC ABGA were significantly lower than those obtained via optical methods. This bias may deserve attention of anesthesiologists when POC ABGA Hb level is used as a transfusion guideline.

Key Words: Blood gas analysis, Hemoglobins, Point-of-care testing.

\section{INTRODUCTION}

Point-of-care (POC) arterial blood gas analysis (ABGA) allows simple and quick testing for the appropriateness of ventilation, the measurements of electrolytes and the level of hemoglobin ( $\mathrm{Hb})$ /hematocrit (Hct). It is also used as a guide- line for transfusion in cases of blood loss during surgeries [1]. However, POC ABGA measures Hb using conductivity, and thus generates different results from measurements utilizing optical methods that are performed in laboratories. A number of studies have reported that hemodilution, cardiopulmonary bypass, and severe hypoalbuminemia influence

This is an Open Access article distributed under the terms of the Creative Commons Attribution Non-Commercial License (http://creativecommons.org/licenses/by-nc/4.0) which permits unrestricted non-commercial use, distribution, and reproduction in any medium, provided the original work is properly cited.

Copyright (c) the Korean Society of Anesthesiologists, 2018 
the $\mathrm{Hb}$ test results of conductivity-based POC ABGA [2-4]. In fact, the authors of this study compared the $\mathrm{Hb} / \mathrm{Hct}$ measurements generated by ABGA in the operating room and those measured in the laboratory and found that ABGA performed in an operating room tends to result in lower values. If such a tendency is true, the misleading results could lead to unnecessary transfusions during operations [4].

The aim of this study was to collect and analyze $\mathrm{Hb}$ data simultaneously measured via POC ABGA in the operating room and in the laboratory to compare the results obtained via two methods for any differences. Furthermore, if there were differences, this study sought to identify measures to reduce such differences.

\section{MATERIALS AND METHODS}

This study was a retrospective study and was conducted following approval from the Institutional Review Board of our Hospital (IRB no. 2016-01-021). We collected test results from patients who underwent surgery between 2015 and 2016. Table 1 shows patients characteristics and data at the time of sampling. We performed arterial blood gas analysis (ABGA) and collected complete blood count (CBC) samples from the radial or dorsalis pedis artery to determine $\mathrm{Hb} / \mathrm{Hct}$ and

Table 1. Demographic Data, Data at the Time of Hemoglobin Analysis

\begin{tabular}{lc}
\hline \multicolumn{1}{c}{ Characteristic } & Value \\
\hline No. of Hb pairs/patients, $\mathrm{n}$ & $86 / 71$ \\
Gender (M/F), $\mathrm{n}$ & $41 / 30$ \\
Age $(\mathrm{yr})$ & $60 \pm 17$ \\
Height $(\mathrm{cm})$ & $162.8 \pm 8.5$ \\
Weight $(\mathrm{kg})$ & $60.7 \pm 12.9$ \\
Blood loss $(\mathrm{ml})$ & $700(500,1,050)$ \\
Fluid administration $(\mathrm{ml})$ & \\
Crystalloid & $1,625(950,2,150)$ \\
Colloid & $0(0,250)$ \\
Transfusion (ml) & $255(0,600)$ \\
Type of surgery & \\
Abdominal & $30(34.9)$ \\
Thoracic & $15(17.4)$ \\
Vascular & $1(1.2)$ \\
Neurosurgical & $7(8.1)$ \\
Orthopedic & $23(26.7)$ \\
Gynecological & $4(4.7)$ \\
Urological & $4(4.7)$ \\
Others & $2(2.3)$ \\
\hline
\end{tabular}

Values are presented as the mean $\pm \mathrm{SD}$, median $(1 \mathrm{Q}, 3 \mathrm{Q})$ or numbers (\%). Hb: hemoglobin. platelet count in patients who underwent surgery and experienced blood loss of more than $500 \mathrm{ml}$. The first $10 \mathrm{ml}$ of blood drawn from the vessel was discarded to exclude dilution. Following collection, samples were obtained and divided into CBC tubes (BD Vacutainer ${ }^{\circledR}$, Becton Dickinson, USA) and $\mathrm{ABGA}$ syringes (BD Preset ${ }^{\mathrm{TM}}$, Becton Dickinson, UK), as directed by each manufacturer. The test results comprised $\mathrm{Hb} / \mathrm{Hct}$ results measured by the Sysmex XE-2100 hematology analyzer (Sysmex Corporation, Japan) in the laboratory and those measured by the GEM Premier 3500 blood gas analyzer (Instrumentation Laboratory, USA) as per POC ABGA in the operating room. Both the Sysmex XE-2100 and the GEM Premier 3500 underwent periodic quality controls (every 6 months and every 3 weeks).

\section{Statistical analysis}

The $\mathrm{Hb}$ value measured by the Sysmex XE-2100 was set as the gold standard and was compared with that measured by the GEM Premier 3500 as per the POC AGBA. The mean bias, standard deviation (SD), 95\% confidence interval (95\% CI), and standard error of the difference of results obtained via the two methods were computed, and Bland-Altman analysis was performed. Evaluation of the clinical significance of the results obtained by Bland-Altman analysis was performed through calculation of the percentage error (\%) based on Critchley and Critchley's formula [5], which doubles the SD of the difference between the two measurements and divides this value by the mean value of the two measurements, as follows:

$$
\text { Percentage error }=(2 \times \mathrm{SD} / \text { mean } \mathrm{Hb}) \times 100 \text {. }
$$

The results of the two methods were considered to be equivalent when the percentage error was $<30 \%$ [5].

The limit of agreement was calculated by: mean $\mathrm{Hb}$ bias $\pm 1.96 \times$ SD. The null hypothesis was set to the difference of the two results being 0 . We conducted a one-sample $t$-test to determine whether the actual difference of results was significantly different from 0 . A 95\% CI was chosen and the null hypothesis was to be rejected when $\mathrm{P}<0.05$, which would indicate that the two values were significantly different.

The clinical utility of the POC AGBA device was tested by indication or contraindication of the transfusion requirement 
using error grid analysis. Zone A should contain $95 \%$ of all measurements and indicates a difference that is clinically acceptable without affecting the transfusion indication. Zone B is defined as the area of significant errors (optimally less than $5 \%$ of all measurements), which is the area between zone A and zone C. Finally, zone $\mathrm{C}$ is defined as the area of major therapeutic errors [6]. The 95\% CI for each zone was calcu-

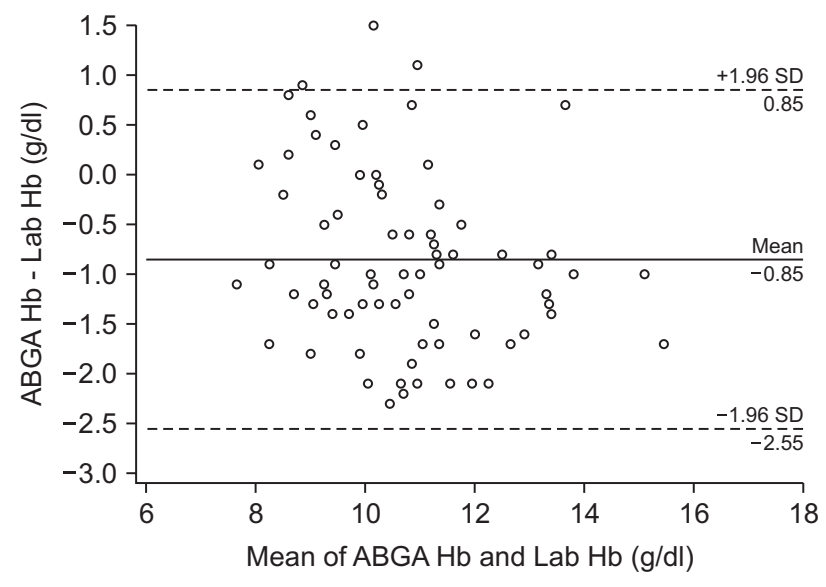

Fig. 1. Bland-Altman plot of difference between ABGA measured hemoglobin and laboratory measured hemoglobin. Solid line: bias $=-0.9 \mathrm{~g} /$ dl, Dashed lines: the upper and lower limits of agreement (1.96 times the SD). ABGA Hb: arterial blood gas analysis measured hemoglobin, Lab Hb: laboratory measured hemoglobin, SD: standard deviation.

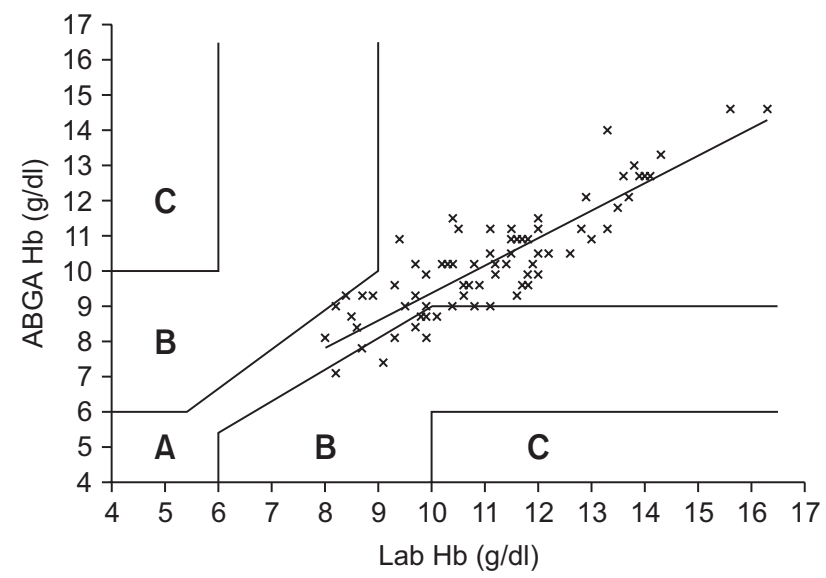

Fig. 2. Paired hemoglobin values provided by laboratory measured hemoglobin and ABGA measured hemoglobin plotted using three zones error grid. Zone A represents the area of clinical acceptable difference, zone B represents the area of significant errors, although their magnitude is not as significant as in zone $C$ which represents the area of major therapeutic errors leading to unnecessary or delayed transfusion. Zone A, B and C encompassed 89.5\% (95\% confidence interval [Cl], 81.1\%-95.1\%) , 10.5\% (95\% Cl, 4.9\%-18.9\%) and 0\% (95\% Cl, $0 \%-4.2 \%)$ of data pairs. ABGA Hb: arterial blood gas analysis measured hemoglobin, Lab Hb: laboratory measured hemoglobin. lated using the Clopper-Pearson exact method [7].

In addition, linear regression analysis was performed to determine the relationship between the $\mathrm{Hb}$ measurement performed via POC ABGA and that performed in the laboratory. The $\mathrm{Hb}$ value obtained via POC ABGA was adjusted as per the regression equation (adjusted $\mathrm{Hb}$ ), and the agreement between the adjusted $\mathrm{Hb}$ and the $\mathrm{Hb}$ value obtained in the laboratory was evaluated. All the statistical analyses were performed using Medcalc software package, version 16.1.2 (MedCalc Software, Belgium) for Windows ${ }^{\circledR}$.

\section{RESULTS}

We collected a total of 86 samples via a chart review. Figs. 1 and 2 show the results of Bland-Altman analysis and error grid analysis that provides the difference between the $\mathrm{Hb}$ values measured via GEM Premier 3500 and those measured via Sysmex XE-2100. Fig. 1 shows the difference in the absolute values of the two measurements; the two values had a bias of $-0.9 \mathrm{~g} / \mathrm{dl}$ ( $\mathrm{P}<0.001,95 \% \mathrm{CI},-1.038$ to -0.665$)$, upper agreement limit of $0.9 \mathrm{~g} / \mathrm{dl}$, lower agreement limit of $-2.6 \mathrm{~g} / \mathrm{dl}$. The correlation coefficient ( $\mathrm{r}$ ) between the two values was 0.88 $(\mathrm{P}<0.001)$, and the percentage error was $16.4 \%$. According to error grid analysis, zones A, B, and C encompassed $89.5 \%$ (95\% CI, 81.1\%-95.1\%), 10.5\% (95\% CI, 4.9\%-18.9\%) and 0\% (95\% CI, 0\%-4.2\%), respectively, of data pairs (Fig. 2). Fig. 3 illustrates the linear regression analysis of the gold standard $\mathrm{Hb}$ and $\mathrm{ABGA} \mathrm{Hb}$ values with the following regression equa-

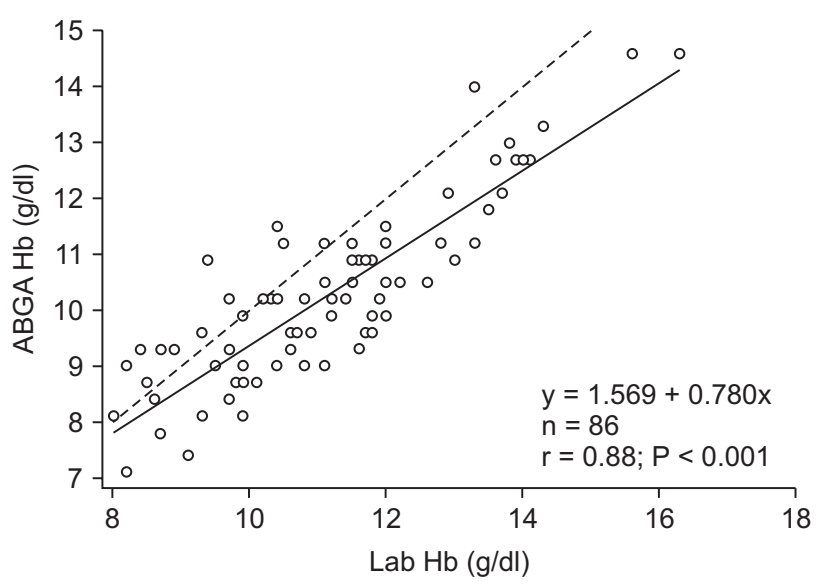

Fig. 3. Scatter plot of the laboratory measured hemoglobin and ABGA measured hemoglobin. Solid line: linear regression graph, Dashed line: $y=x$. ABGA Hb: arterial blood gas analysis measured hemoglobin, Lab $\mathrm{Hb}$ : laboratory measured hemoglobin. 
tion, as follows:

$\mathrm{y}=1.569+0.780 \mathrm{x}(\mathrm{x}$ : Laboratory measured Hb; y: ABGA measured $\mathrm{Hb})$.

The ABGA $\mathrm{Hb}$ value was adjusted using this equation. We subtracted 1.569 from the ABGA $\mathrm{Hb}$ and divided it by 0.780 to obtain the adjusted Hb value. Figs. 4 and 5 show the difference in the adjusted $\mathrm{Hb}$ values and the gold standard $\mathrm{Hb}$ as shown via the Bland-Altman analysis and error grid analysis.

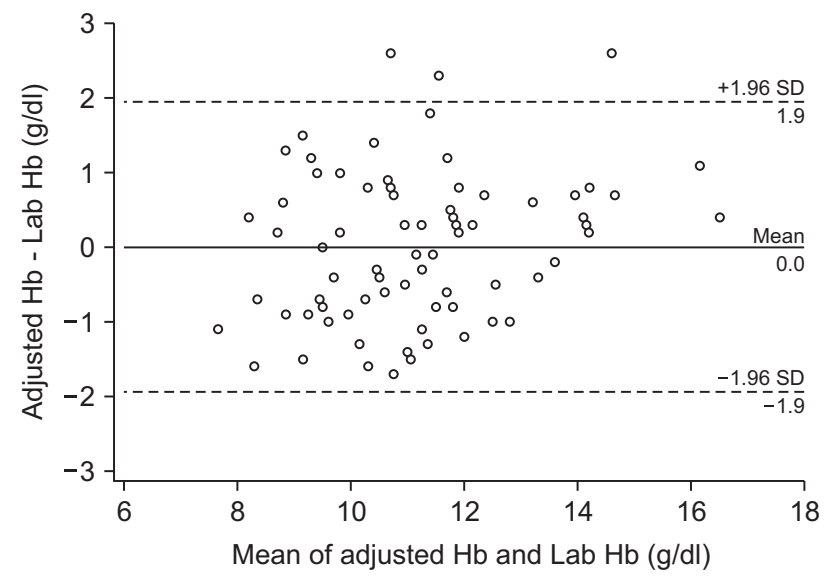

Fig. 4. Bland-Altman plot of difference between adjusted hemoglobin and laboratory measured hemoglobin. Solid line: bias $=0 \mathrm{~g} / \mathrm{dl}$, Dashed line: the upper and lower limits of agreement (1.96 times the SD). Adjusted $\mathrm{Hb}$ : hemoglobin adjusted by linear regression graph, Lab $\mathrm{Hb}$ : laboratory measured hemoglobin, SD: standard deviation.

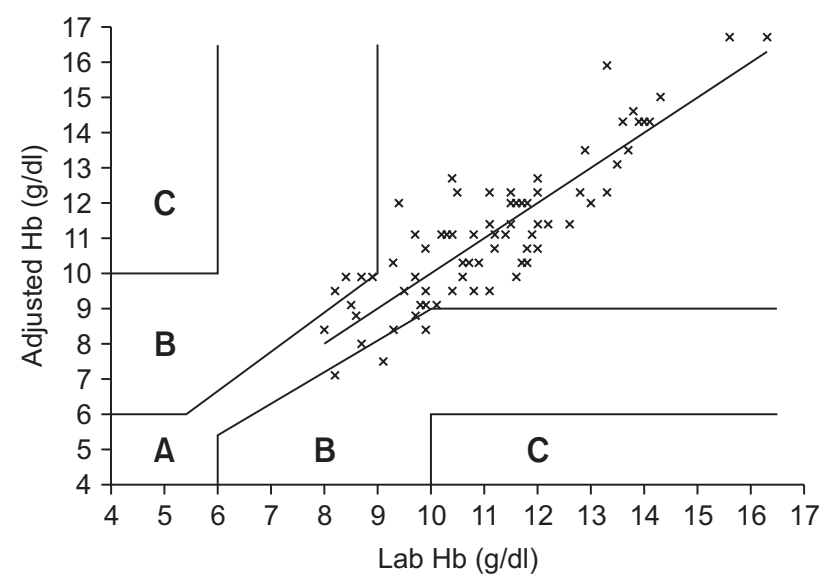

Fig. 5. Paired hemoglobin values provided by laboratory hemoglobin and adjusted hemoglobin plotted using three zones error grid. Zone A, $\mathrm{B}$ and $\mathrm{C}$ encompassed 91.9\% (95\% confidence interval [Cl], 83.9\%96.7\%) , 8.1\% (95\% Cl, 3.3\%-16.1\%) and 0\% (95\% Cl, 0\%-4.2\%) of data pairs. Adjusted $\mathrm{Hb}$ : hemoglobin adjusted by linear regression graph, Lab Hb: laboratory measured hemoglobin.
Fig. 4 shows the difference in the absolute values of the two measurements with a bias of $0 \mathrm{~g} / \mathrm{dl}(\mathrm{P}=0.991)$, upper agreement limit of $1.9 \mathrm{~g} / \mathrm{dl}$, lower agreement limit of $-1.9 \mathrm{~g} / \mathrm{dl}$. The correlation coefficient (r) between the two values was 0.88 $(\mathrm{P}<0.001)$, with a percentage error of $18.2 \%$. Fig. 5 shows the three-zone error grid; zones A, B, and C encompassed $91.9 \%$ (95\% CI, 83.9\%-96.7\%), 8.1\% (95\% CI, 3.3\%-16.1\%) and 0\% (95\% CI, 0\%-4.2\%), respectively, of data pairs. Table 2 summarizes the absolute value of the difference between laboratory measured $\mathrm{Hb}$ and $\mathrm{ABGA}$ POC measured $\mathrm{Hb}$ or adjusted $\mathrm{Hb}$. The percentage of the calculated difference within $1.0 \mathrm{~g} /$ $\mathrm{dl}$ between laboratory $\mathrm{Hb}$ and ABGA $\mathrm{Hb}$ was $51.2 \%$, and after adjusted regression, the percentage of the difference within $1.0 \mathrm{~g} / \mathrm{dl}$ between laboratory $\mathrm{Hb}$ and adjusted $\mathrm{Hb}$ was $72.1 \%$.

\section{DISCUSSION}

The result of the Bland-Altman analysis between the laboratory $\mathrm{Hb}$ and $\mathrm{ABGA} \mathrm{Hb}$ values showed that the mean bias was $-0.9 \mathrm{~g} / \mathrm{dl}$ with a percentage error of $16.4 \%$, indicating a significant difference between the two values. However, a Bland-Altman analysis comparing the adjusted $\mathrm{Hb}$ value that was obtained via a linear regression analysis and the laboratory $\mathrm{Hb}$ value showed that there was no significant difference between these two values. The percentage error after adjustment was $18.2 \%$. The reliability of the transfusion decision was assessed using the three-zone error grid. According to Morey et al. [6], zone B should encompass less than $5 \%$ of data pairs. However, $10.5 \%$ of original data pairs and $8.1 \%$ of adjusted data pairs were included in zone B in the present study. Thus, the use of adjusted Hb values instead of ABGA

Table 2. Absolute Values of the Difference between Laboratory Measured Hemoglobin and ABGA Measured Hemoglobin or Adjusted Hemoglobin

\begin{tabular}{ccc}
\hline $\begin{array}{c}\text { Difference from } \\
\text { Lab Hb value }\end{array}$ & Lab Hb-ABGA Hb & Lab Hb-Adjusted Hb \\
\hline$<1.0 \mathrm{~g} / \mathrm{dl}$ & $44(51.2)$ & $62(72.1)$ \\
$>1.0 \mathrm{~g} / \mathrm{dl}$ & $42(48.8)$ & $24(27.9)$ \\
\hline
\end{tabular}

Values are presented as number (\%). Lab $\mathrm{Hb}$ : laboratory measured hemoglobin, ABGA Hb: arterial blood gas analysis measured hemoglobin, Adjusted $\mathrm{Hb}$ : hemoglobin adjusted by linear regression. Regression equation between laboratory measured $\mathrm{Hb}$ and $\mathrm{ABGA}$ measured $\mathrm{Hb}$ : $\mathrm{y}$ $=1.569+0.780 x(x$ : Laboratory measured $\mathrm{Hb}$; $\mathrm{y}$ : ABGA measured $\mathrm{Hb})$. Adjusted $\mathrm{Hb}=($ ABGA Hb - 1.569) $/ 0.780$. 
$\mathrm{Hb}$ values is questionable.

The Sysmex XE-2100, which is used for $\mathrm{Hb} / \mathrm{Hct}$ testing in laboratories, uses a sulfolyser to denature $\mathrm{Hb}$, converts it into sodium lauryl sulfate hemoglobin by structurally changing the globin and oxidizing the heme, and measures the absorbance. For this test, $3 \mathrm{ml}$ of blood must be injected into the ethylenediaminetetraacetic acid test tube, and the entire test takes about 30 minutes. The GEM Premier 3500, which is used for POC ABGA in operating rooms, measures Hct by its conductivity; a small amount of a whole blood sample (135 $\mu \mathrm{l}$ ) is tested via aspiration within the disposable cartridge that comprises testing reagent and minimized sensor, and the entire test takes about 85 seconds, which is a shorter length of time than the laboratory test [8]. The $\mathrm{Hb} / \mathrm{Hct}$ is measured via the following principle: when a low-frequency current is incurred on the blood sample, the non-conductive cell membranes of red blood cells cannot conduct the current, meaning that a higher number of red blood cells results in a lower conductivity; such difference in conductivities is used to measure the $\mathrm{Hb} / \mathrm{Hct}[2,9]$. Again, the entire test requires only a few minutes, which makes POC ABGA useful in situations where simple and quick testing is demanded, such as for patients during a surgery [10].

In most surgeries, crystalloid solutions are usually infused before the induction of anesthesia to prevent any anesthesiainduced hypotension, which results in hemodilution [11]. When such crystalloid solutions, which are abundant with electrolytes, lead to hemodilution, the $\mathrm{Hb} / \mathrm{Hct}$ measurements that are taken by conductivity-based POC ABGA can be lower than the usual values; the difference in values tends to increase with lower Hct levels $[4,10,11]$. The Hb/Hct measurements are lower than the gold standard values obtained via optical methods because an increased concentration of electrolytes increases plasma conductivity $[1,2]$. As predicted, the $\mathrm{Hb}$ measurements taken with the GEM Premier 3500 in this study were also lower than the $\mathrm{Hb}$ values obtained with the Sysmex XE-2100 (difference of values: $-0.9 \mathrm{~g} / \mathrm{dl}$ ). This comparison revealed that conductivity-based POC testing methods for patients with hemodilution induced by crystalloid solutions show a downward bias compared to measurements taken via optical methods.

Unlike crystalloid solutions, colloid solutions tend to induce an upward bias in the $\mathrm{Hb} / \mathrm{Hct}$ measurements performed via POC ABGA compared to those performed in the laboratory [2]. This is because administration of colloid solutions during a conductivity-based POC ABGA reduces plasma conductivity, which reduces the overall blood conductivity, leading to increased Hct measurements. Furthermore, the high viscosity of colloid solutions is also known to increase Hct measurements obtained by POC ABGA [1,2].

Our adjustment method using a regression equation can be easily adapted to the GEM Premier 3500, decreasing downward bias in data pairs in zone B. Furthermore, the percentage of the calculated difference within $1.0 \mathrm{~g} / \mathrm{dl}$ between laboratory $\mathrm{Hb}$ and $\mathrm{ABGA} \mathrm{Hb}$ was $51.2 \%$; however, after adjusted regression, the percentage of the difference within $1.0 \mathrm{~g} / \mathrm{dl}$ between laboratory $\mathrm{Hb}$ and adjusted $\mathrm{Hb}$ increased to $72.1 \%$.

This study was conducted via a retrospective chart review and is bound to have some limitations. The composition of the patient sample was not considered, and variables such as the types of surgery, types and doses of administered fluids, injection of albumin, and transfusion were not controlled. Nevertheless, the authors of this study postulated that the employed method was valid for the objective of the current study, which was to verify whether $\mathrm{Hb} /$ Hct measurements obtained by POC ABGA could be used as an appropriate transfusion guideline in general operating rooms regardless of the type of surgery being performed as well as in specific surgical situations. At a minimum, our findings suggest that interpretation of $\mathrm{Hb} / \mathrm{Hct}$ values obtained via POC ABGA in the operating room as a transfusion guideline should be undertaken with caution.

When we adjusted the ABGA results via a linear regression analysis, we found that the percentage of error of the adjusted $\mathrm{Hb}(18.2 \%)$ was $1.8 \%$ greater than the value before adjustment (16.4\%). Both values had a percentage error of less than $30 \%$, which signifies agreement with the $\mathrm{Hb}$ values obtained in the laboratory; however, because the percentage error actually increased after adjustment of the value, a search for other methods to adjust $\mathrm{Hb}$ values may be necessary.

In conclusion, $\mathrm{Hb}$ measurements obtained with reference to conductivity via a POC ABGA were significantly lower than those obtained via optical methods in laboratories. Hence, caution should be used in interpreting the conductivitybased POC ABGA results as a transfusion guide, in order to prevent unnecessary transfusions. In addition, although the downward bias of the $\mathrm{Hb}$ measurements obtained via POC 
ABGA was adjusted using a regression equation, the percentage error of the adjusted value actually increased; thus, there should be more discussions on the appropriate method of adjustment and the need for adjustment. We also expect the development of a POC ABGA device capable of reflecting the downward bias of POC ABGA Hb values to obtain more accurate $\mathrm{Hb}$ values.

\section{REFERENCES}

1. Wu P, Morey TE, Harris NS, Gravenstein N, Rice MJ. Intravenous fluids cause systemic bias in a conductivity-based point-of-care hematocrit meter. Anesth Analg 2012; 114: 314-21.

2. Steinfelder-Visscher J, Weerwind PW, Teerenstra S, Pop GA, Brouwer RM. Conductivity-based hematocrit measurement during cardiopulmonary bypass. J Clin Monit Comput 2007; 21: 7-12.

3. Hopfer SM, Nadeau FL, Sundra M, Makowski GS. Effect of protein on hemoglobin and hematocrit assays with a conductivitybased point-of-care testing device: comparison with optical methods. Ann Clin Lab Sci 2004; 34: 75-82.
4. Myers GJ, Browne J. Point of care hematocrit and hemoglobin in cardiac surgery: a review. Perfusion 2007; 22: 179-83.

5. Critchley LA, Critchley JA. A meta-analysis of studies using bias and precision statistics to compare cardiac output measurement techniques. J Clin Monit Comput 1999; 15: 85-91.

6. Morey TE, Gravenstein N, Rice MJ. Let's think clinically instead of mathematically about device accuracy. Anesth Analg 2011; 113: 89-91.

7. Brown LD, Cai TT, DasGupta A. Interval estimation for a binomial proportion. Statist Sci 2001; 16: 101-33.

8. Bénéteau-Burnat B, Bocque MC, Lorin A, Martin C, Vaubourdolle M. Evaluation of the blood gas analyzer Gem PREMIER 3000. Clin Chem Lab Med 2004; 42: 96-101.

9. Visser KR. Electric conductivity of stationary and flowing human blood at low frequencies. Med Biol Eng Comput 1992; 30: 63640.

10. Steinfelder-Visscher J, Weerwind PW, Teerenstra S, Brouwer MH. Reliability of point-of-care hematocrit, blood gas, electrolyte, lactate and glucose measurement during cardiopulmonary bypass. Perfusion 2006; 21: 33-7.

11. Garrioch SS, Gillies MA. Which intravenous fluid for the surgical patient? Curr Opin Crit Care 2015; 21: 358-63. 